\title{
RNAi-based functional elucidation of PtrPRP, a gene encoding a hybrid proline rich protein, in cold tolerance of Poncirus trifoliata
}

\author{
Ting Peng ${ }^{1,2}$, Mao-Mao Jia ${ }^{1}$ and Ji-Hong Liu' ${ }^{1 *}$
}

${ }^{1}$ Key Laboratory of Horticultural Plant Biology, College of Horticulture and Forestry Sciences, Huazhong Agricultural University, Wuhan, China, ${ }^{2}$ National Navel Orange Engineering Research Center, College of Navel Orange, Gannan Normal University, Ganzhou, China

\section{OPEN ACCESS}

Edited by:

Zhulong Chan,

Wuhan Botanic Garden, Chinese Academy of Sciences, China

Reviewed by:

Charles L. Guy,

University of Florida, USA

Avi Sadka,

Agricultural Research Organization,

Israel

*Correspondence:

Ji-Hong Liu,

Key Laboratory of Horticultural Plant

Biology, College of Horticulture and Forestry Sciences, Huazhong

Agricultural University,

Wuhan 430070, China

liujihong@mail.hzau.edu.cn

Specialty section:

This article was submitted to

Plant Physiology,

a section of the journal

Frontiers in Plant Science

Received: 31 July 2015 Accepted: 16 September 2015 Published: 29 September 2015

Citation:

Peng T, Jia M-M and Liu J-H (2015)

RNAi-based functional elucidation of PtrPRP, a gene encoding a hybrid proline rich protein, in cold tolerance

of Poncirus trifoliata.

Front. Plant Sci. 6:808

doi: 10.3389/fpls.2015.00808
Hybrid proline-rich proteins (HyPRPs) have been suggested to play important roles in various plant development and stress response. In this study, we report the cloning and functional analysis of PtrPRP, a HyPRP-encoding gene of Poncirus trifoliata. PtrPRP contains 176 amino acids, among which 21\% are proline residues, and has an 8-cysteine motif (8 CM) domain at the $\mathrm{C}$ terminal, a signal peptide and a proline-rich region at the $\mathrm{N}$ terminal. PtrPRP is constitutively expressed in root, stem and leaf, with the highest expression levels in leaf. It was progressively induced by cold, but transiently upregulated by salt and ABA. Transgenic $P$. trifoliata plants with knock-down PtrPRP by RNA interference (RNAi) were generated to investigate the role of PtrPRP in cold tolerance. When challenged by low temperature, the PtrPRP-RNAi plants displayed more sensitive performance compared with wild type (WT), as shown by higher electrolyte leakage and malondialdehyde content. In addition, the RNAi lines accumulated more reactive oxygen species (ROS) and lower levels of proline relative to WT. These results suggested that PtrPRP might be positively involved in cold tolerance by maintaining membrane integrity and ROS homeostasis.

\section{Keywords: cold stress, Poncirus trifoliata, hybrid proline-rich protein, RNA interference, ROS}

\section{Introduction}

Plants are frequently challenged with a variety of abiotic stresses, among which cold, at either freezing, or chilling regimes, constitutes an important factor leading to adverse impacts on plant growth, development and yield potential. Chilling and freezing stresses lead to physiological or structural alterations, such as elevation of reactive oxygen species (ROS), imbalance of osmotic pressure, and formation of ice crystals. All of these unfavorable situations will result in deterioration of membrane integrity, impairment of cell viability and eventually lead to cell death when they are not precisely coped with (Xiong and Zhu, 2002; Einset et al., 2007; Peng et al., 2012).

It has been well known that to survive under cold plants experience a cascade of physiological and biochemical changes, such as accumulation of various proteins or soluble compounds, and alteration of a series of metabolic reactions (Wilson and Cooper, 1994; Knight et al., 1996; Fowler et al., 1999; Welling and Palva, 2006; Huang et al., 2011). 
In addition, accumulating evidences show that extensive reprogramming of a cohort of cold-responsive genes is an elegant strategy for the plants to adapt to the harsh environments (Stockinger et al., 1997; Zarka et al., 2003; Nakashima and Yamaguchi-Shinozaki, 2006; Shi et al., 2015). These genes are generally classified into two major categories, functional proteins, and regulatory proteins, which play either direct protective or regulatory roles in stress tolerance (Yamaguchi-Shinozaki and Shinozaki, 2005). For example, the functional proteins participate in stabilization of membrane integrity, maintenance of enzyme activity, physical structures of cellular components, which are critical factors contributing to enhance stress tolerance in plants (Walker et al., 2010). Genetic manipulation of the coldresponsive genes has been suggested to serve as an alternative approach for generating transgenic plants with enhanced stress tolerance (Huang et al., 2013). However, it is worth mentioning that functional characterization of a stress-responsive gene is required before it can be efficiently manipulated.

Hybrid proline-rich proteins (HyPRPs) are a subset of prolinerich proteins (PRPs) specific to seed plants; however, they do not contain the domain typical of PRPs (Josè-Estanyol et al., 2004; Priyanka et al., 2010; Xu et al., 2011). Based on the position and number of cysteine residues at $\mathrm{C}$ terminal, HrPRPs can be categorized into two major groups, in which Class $\mathrm{A}$ has 4-6 cysteine residues, while Class B contains a conserved eight-cysteine-motif ( $8 \mathrm{CM})$ at $\mathrm{C}$ terminal, a repetitive prolinerich N-terminal domain (PRD) and a signal peptide in front of the PRD (Josè-Estanyol and Puigdomènech, 2000; JosèEstanyol et al., 2004; Battaglia et al., 2007; Dvořáková et al., 2007; Neto et al., 2013). HyPRPs have been suggested to play important biological roles in various processes, including plant cell elongation, ontogeny, and morphogenesis of different organs, defenses against viral or fungal pathogens (He et al., 2002; Holk et al., 2002; Dvořáková et al., 2012). In addition, HyPRPs of Arabidopsis thaliana, Brassica napus, Medicago sativa, Glycine max, and M. truncatula have been also suggested to be involved in responses to biotic and abiotic stresses (Deutch and Winicov, 1995; He et al., 2002; Bouton et al., 2005; Zhang and Schläppi, 2007). Nevertheless, so far little knowledge is available on the role of HyPRPs in stress tolerance of perennial plants, such as Poncirus trifoliata.

Poncirus trifoliata (L.) Raf. is extremely cold hardy when it is fully cold acclimated. In a previous work, suppression subtractive hybridization was employed to unravel cold-responsive genes of valuable significance for engineering cold tolerance (Peng et al., 2012). One of the ESTs draws our attention as its expression level was elevated over 70-folds under cold; the EST was later annotated to encode a HyPRP. However, whether it plays a role in cold tolerance remains undetermined. As a follow-up and continuum of our earlier work, in this study we report the isolation and functional analysis of this gene, designated as PtrPRP, in cold tolerance by RNA interference (RNAi). Transcript levels of PtrPRP were enhanced by abiotic stresses, but the response to cold was extremely dramatic. Knockdown of PtrPRP in trifoliate orange by RNAi led to enhanced cold sensitivity. We further demonstrated that the RNAi lines accumulated more ROS and malondialdehyde (MDA), but lower levels of proline. Our data indicate that PtrPRP is a coldresponsive gene that plays an essential role in cold tolerance.

\section{Materials and Methods}

\section{Plant Materials and Stress Treatments}

Three-month-old trifoliate orange seedlings were grown in a growth chamber $\left(25^{\circ} \mathrm{C}\right)$ with a photoperiod regime of $8 \mathrm{~h}$ dark/16 $\mathrm{h}$ light (light intensity is about $100 \mu \mathrm{mol} \mathrm{m} \mathrm{m}^{-2} \mathrm{~s}$ ), and an relative humidity of $60-70 \%$. The seedlings were well watered before they were subjected to various stresses. For cold treatment the seedlings were placed in the chamber set at $4^{\circ} \mathrm{C}$ for $6 \mathrm{~d}$, followed by transfer to $25^{\circ} \mathrm{C}$ for recovery. The leaves were sampled at $0,6,24,72$, and $144 \mathrm{~h}$ after cold treatment and $6 \mathrm{~h}$ after recovery. Dehydration was imposed by placing the seedlings on a clean bench under ambient environment for $6 \mathrm{~h}$ and then shifted to water for $0.5 \mathrm{~h}$. The leaves were collected at $0,0.5,1,3$, and $6 \mathrm{~h}$ after dehydration and $6 \mathrm{~h}$ after rehydration. For salt stress, the seedlings were treated with $200 \mathrm{mM} \mathrm{NaCl}$ solution for up to $144 \mathrm{~h}$ and then shifted to water for another $6 \mathrm{~h}$; the leaves were sampled at $0,6,24,72$, and $144 \mathrm{~h}$ of salt treatment and after $6 \mathrm{~h}$ of recovery in water. In addition, the seedlings were treated with $100 \mu \mathrm{M}$ ABA for $0,6,12,24 \mathrm{~h}$, and $48 \mathrm{~h}$, followed by transfer to water for another $6 \mathrm{~h}$; the leaves were sampled at the designated time points. The samples were immediately frozen in liquid nitrogen and stored at $-80^{\circ} \mathrm{C}$ until further use.

\section{Gene Isolation and Sequence Analysis}

Rapid amplification of cDNA ends (RACEs) was employed to obtain full-length cDNA of PtrPRP. For this purpose, genespecific primers for $5^{\prime}$-RACE and $3^{\prime}$-RACE (Table 1) were designed based on the EST sequence identified in the previous work (Peng et al., 2012). RACE-PCR was carried out using the SMART $^{\mathrm{TM}}$ RACE cDNA Kit (Clontech, USA). Total RNA was

\section{TABLE 1 | Oligonucleotide primers used in this study.}

\begin{tabular}{ll}
\hline Name & Primer sequences $\left(\mathbf{5}^{\prime} \mathbf{-} \mathbf{3}^{\prime} \mathbf{)}\right.$ \\
\hline $5^{\prime}$-RACE & CGCCAAACCGAAATGTGCTCTGATA \\
3'-RACE & TAGTGTGAGATACCCACCGCC \\
PtrPRP-S & ATGGGAAAATATCAATTAGC \\
PtrPRP-A & TTAAGCAGGACACTGAAATCC \\
PtrActin-S & CATCCCTCAGCACCTTCC \\
PtrActin-A & CCAACCTTAGCACTTCTCC \\
PtrPRP-q-S & ACCGATTGTAAAGACGCCAC \\
PtrPRP-q-A & CACCGAGTTGAGAGCATCA \\
PtrPRP-L-S & GAAGATCTATGGGAAAATATCAATTAGC \\
PtrPRP-L-A & GACTAGTAGCAGGACACTGAAATCC \\
PtrPRP-attB-S & GGGACAAGTTGTACAAAAAAGCAG \\
PtrPRP-attB-A & GCTATGGGAAAATATCAATAGC \\
GGGGACCACTTGTACAAGAAAGCT & GGGTCTTAGGGCTTGGTCCGTTA \\
NPT II-A & AGACAATCGGCTGCTCTGAT \\
PtrPRP-s-S & TCATTCGAACCCCAGAGTC \\
PtrPRP-s-A & ATGGGAAAATATCAATTAGC \\
& TAAGCAGGACACTGAAATCC
\end{tabular}


extracted with RNAiso Plus (TaKaRa, Japan) according to the manufacturer's instructions. First strand cDNA was synthesized via PrimeScript ${ }^{\circledR}$ RT Reagent Kit With gDNA Eraser (TaKaRa) following the user's manual. The $5^{\prime}$-RACE and $3^{\prime}$-RACE PCR products were sequenced and analyzed, and then merged with the original EST to get a single sequence, which was verified using RT-PCR with a pair of full-length primers (PtrPRP-S/PtrPRPA, Table 1). Molecular weight (MW) and isoelectric point (pI) of the protein were predicted on ExPASy ${ }^{1}$. Phylogenetic analysis was constructed by Phylogeny.fr online software ${ }^{2}$ (Dereeper et al., 2008) and MEGA4.0. Multiple alignments of the HyPRPs were performed by ClustalX program with defaulted settings and displayed by Jalview ${ }^{3}$. Signal peptide was predicted with SignalP4.1 Server ${ }^{4}$ and iPSORT ${ }^{5}$ (Bannai et al., 2002).

\section{Gene Expression Analysis by Quantitative Real-time RT-PCR (qPCR)}

Total RNA extraction and cDNA synthesis were performed as mentioned above. Expression of PtrPRP under the stresses and in different tissues was assessed by $\mathrm{qPCR}$, which was carried out with the SYBR ${ }^{\circledR}$ Green PCR kit (TaKaRa) on a LightCycler 480 Real-Time System (Roche). PCR solution, in a total volume of $10 \mu \mathrm{l}$, contained $5 \mu \mathrm{l}$ of $2 \times$ SYBR Premix Ex Taq (Tli RNaseH Plus), $50 \mathrm{ng}$ of cDNA, $0.25 \mu \mathrm{M}$ of each primer (PtrPRP-q-S/A). The reaction cycles were $95^{\circ} \mathrm{C}$ for $30 \mathrm{~s}$, and 40 cycles of $95^{\circ} \mathrm{C}$ for $5 \mathrm{~s}, 56^{\circ} \mathrm{C}$ for $10 \mathrm{~s}$, and $72^{\circ} \mathrm{C}$ for $15 \mathrm{~s}$. Each reaction was repeated at least three times, and ${ }^{\Delta \Delta} \mathrm{CT}$ method was applied to calculate relative expression levels. The Actin gene of trifoliate orange was used as a reference control and analyzed in parallel with specific primers (Table 1) to normalize the expression levels.

\section{Subcellular Localization of PtrPRP}

To determine subcellular localization of PtrPRP, the full-length PtrPRP cDNA without stop codon was amplified using primers (PtrPRP-L-S/A) containing restriction sites of BglII and SpeI. The PCR products were purified with AxyPrep ${ }^{\text {TM }}$ DNA Gel Extraction Kit (Axygen scientific, USA), digested with BglII and SpeI and subcloned into the pCAMBIA1302 vector containing a GFP reporter gene, under the control of $\mathrm{CaMV} 35 \mathrm{~S}$ promoter. The resultant fusion construct PtrPRP::GFP and the control vector (GFP) were separately introduced into onion epidermis via Agrobacterium-mediated transformation as described by Peng et al. (2014), followed by visualization of green fluorescence under a confocal microscope (FV1000; Olympus,Tokyo, Japan) or a fluorescence microscope (Nikon 90i).

\section{Generation and Identification of RNAi Plants}

To generate PtrPRP-RNAi plants, a 253-bp cDNA fragment of PtrPRP was amplified using a pair of primers (PtrPRPattB-S/A) and introduced into pHELLSGATE2 through BP recombination reactions (Invitrogen, Japan). The RNAi

\footnotetext{
${ }^{1}$ http://us.expasy.org

${ }^{2}$ http://phylogeny.lirmm.fr/phylo_cgi/index.cgi

${ }^{3}$ http://www.jalview.org/

${ }^{4}$ http://www.cbs.dtu.dk/services/SignalP/

${ }^{5}$ http://hc.ims.u-tokyo.ac.jp/iPSORT/
}

vector was introduced into A. tumefaciens strain GV3101. Agrobacterium-mediated transformation of trifoliate orange was performed according to Fu et al. (2011). Kanamycinresistant shoots were identified by genomic PCR using primers specific to neomycin phosphotransferase II (NPTII-S/A) and the 253-bp sequence (Table 1). Examination of PtrPRP expression was carried out by semi-quantitative RT-PCR according to Shi et al. (2010) except using specific primers (PtrPRP-s-S/A, Table 1). QRT-PCR was also used to confirm the expression of one transgenic line, as done mentioned above. Actin gene was used as the reference gene. The positive transgenic plants were vegetatively propagated to obtain enough plants that were used for the subsequent experiments.

\section{Cold Tolerance Assessment and Physiological Measurements}

Uniform and healthy 3-month-old plants of wild type (WT) and two RNAi lines were used for cold treatment. Two days after sufficient watering, the plants were placed in a growth chamber set at $0^{\circ} \mathrm{C}$ and kept for $48 \mathrm{~h}$ without light in order to avoid light-induced oxidative stress under cold treatment. The leaves were sampled after completion of the chilling treatment and used for measurement of electrolyte leakage (EL), MDA, ROS, and proline.

Measurement of EL was conducted according to Peng et al. (2012). In brief, the sampled leaves were immersed in $20 \mathrm{~mL}$ of double distilled water $\left(\mathrm{ddH}_{2} \mathrm{O}\right)$, while the control tube contained only $20 \mathrm{~mL}$ of $\mathrm{ddH}_{2} \mathrm{O}$. The tubes were gently shaken for $2 \mathrm{~h}$ on a shaker (QB-206, Qilinbeier, China) at room temperature; the EL of sample $\left(C_{1}\right)$ and control $\left(C_{1}\right)$ were then measured on a DSS-307 conductivity meter (SPSIC, China). The tubes were then boiled for $10 \mathrm{~min}$, and cooled down at room temperature before measurement of the $\mathrm{EL}\left(\mathrm{C}_{2}\right.$ and $\left.\mathrm{CK}_{2}\right)$. A relative conductance was calculated by $\mathrm{C}(\%)=\left(\mathrm{C}_{1}-\mathrm{CK}_{1}\right) /\left(\mathrm{C}_{2}-\mathrm{CK}_{2}\right) \times 100$.

Proline content was determined according to Zhao et al. (2009) with minor modification. The leaf tissues (about $0.5 \mathrm{~g}$ ) were extracted in $5 \mathrm{~mL}$ of $3 \%$ sulphosalicylic acid at $95^{\circ} \mathrm{C}$ for $10 \mathrm{~min}$. After cooling down, the homogenate was filtered and $2 \mathrm{~mL}$ of supernatant was transferred to a new tube containing $2 \mathrm{~mL}$ of acetic acid and $2 \mathrm{~mL}$ of acidified ninhydrin reagent. After $30 \mathrm{~min}$ of incubation in boiling water, $4 \mathrm{~mL}$ of toluene was added to the tubes and vortexed for $30 \mathrm{~s}$. The absorbance of the toluene layer was colorimetrically determined at $520 \mathrm{~nm}$. Protein concentration was determined based on the method reported by Bradford (1976).

Accumulation of $\mathrm{H}_{2} \mathrm{O}_{2}$ and $\mathrm{O}_{2}{ }^{-}$, two major types of ROS, was assayed using histochemical staining with $3,3^{\prime}$ diaminobenzidine $(\mathrm{DAB})$ and nitrotetrazolium blue chloride (NBT), respectively. The leaves sampled after $48 \mathrm{~h}$ of chilling treatment were immediately immersed in $1 \mathrm{mg} \mathrm{mL}^{-1}$ freshly prepared NBT or DAB solution at ambient temperature, until blue or brown precipitates were observed. The stained leaves were then bleached in concentrated ethanol, and kept in 70\% ethanol. Quantitative measurement of $\mathrm{H}_{2} \mathrm{O}_{2}$ was also carried out using a specific detection kit based on the manufacturer's instructions (Nanjing Jiancheng Bioengineering Institute, China). 
Malondialdehyde content, expressed as nmol/mg protein, was measured using a detection kit specifically designed for MDA quantification (Nanjing Jiancheng Bioengineering Institute, China) based on the manufacturer's instructions. Protein concentration was determined based on the method reported by Bradford (1976).

\section{Statistical Analysis}

The data were analyzed using analysis of variance (ANOVA), and statistical difference between WT and transgenic lines was compared, taking $P<0.05$ as significant.

\section{Results}

\section{Isolation of PtrPRP from $P$. trifoliata}

In a previous work, an EST annotated as PRP was fished out by SSH-based screening of a cold-treated cDNA library of P. trifoliata (Peng et al., 2012). As the whole genome sequence information of $P$. trifoliata is unavailable at this time, we employed RACE to isolate the full-length cDNA of this gene. For this purpose, $5^{\prime}$-RACE and $3^{\prime}$-RACE PCR were carried out using primers designed base on the original EST, leading to amplification of two fragments of 554 and 802 bp, respectively, which were shown to be homologous to known PRP genes by
Blastn against NCBI. Assembly of the two RACE sequences and the original EST resulted in generation of a full-length sequence of 849 bp in size, which was further verified by PCR to be correct in the sequence. The sequence displayed $74 \%$ of identity to PRP gene of Solanum palustre; so the gene was designated as PtrPRP ( Poncirus trifoliata $\underline{\text { Proline-Rich } \underline{\text { Protein }) . ~ T h e ~ g e n e ~ h a s ~}}$ been deposited in the NCBI database under the accession number of KF171887.1.

\section{Sequence Analysis of PtrPRP}

The full-length PtrPRP cDNA contained a 531-bp open reading frame (ORF) encoding 176 amino acids, 21\% of which were proline residues (Figure 1). PtrPRP had a predicted molecular mass of $18.242 \mathrm{kD}$ and an isoelectric point of 8.65. In addition, a signal peptide composed of 24 amino acids was observed at the $\mathrm{N}$ terminal. In order to reveal the relationship between PtrPRR and PRP proteins from other plants, we constructed a phylogenetic tree using deduced amino acid sequences of PtrPRP and PRPs from other plants. PRPs were evolutionarily divergent in plant. However, PtrPRP was not clustered into the five groups that have been established by earlier studies (data not shown). Therefore, we assume that PtrPRP might be a HyPRP. Alignment with PtrPRP and HyPRPs from Phaseolous vulgaris, A. thaliana, G. max, Nicotiana tabacum, and Capsicum annuum revealed that PtrPRP has the conserved $8 \mathrm{CM}$ domain at the $\mathrm{C}$

\section{PtrPRP/1-176 \\ AAC49369/1-127 \\ AAM63191.1/1-111 \\ AAM75351.1/1-179 \\ BAA95941/1-158 \\ ABQ88334/1-142}

PtrPRP/1-176 AAC49369/1-127 AAM63191.1/1-111 AAM75351.1/1-179 BAA95941/1-158 ABQ88334/1-142

PtrPRP/1-176 AAC49369/1-127 AAM63191.1/1-111 AAM75351.1/1-179 BAA95941/1-158 ABQ88334/1-142

PtrPRP/1-176 AAC49369/1-127 AAM63191.1/1-111 AAM75351.1/1-179 BAA95941/1-158 ABQ88334/1-142
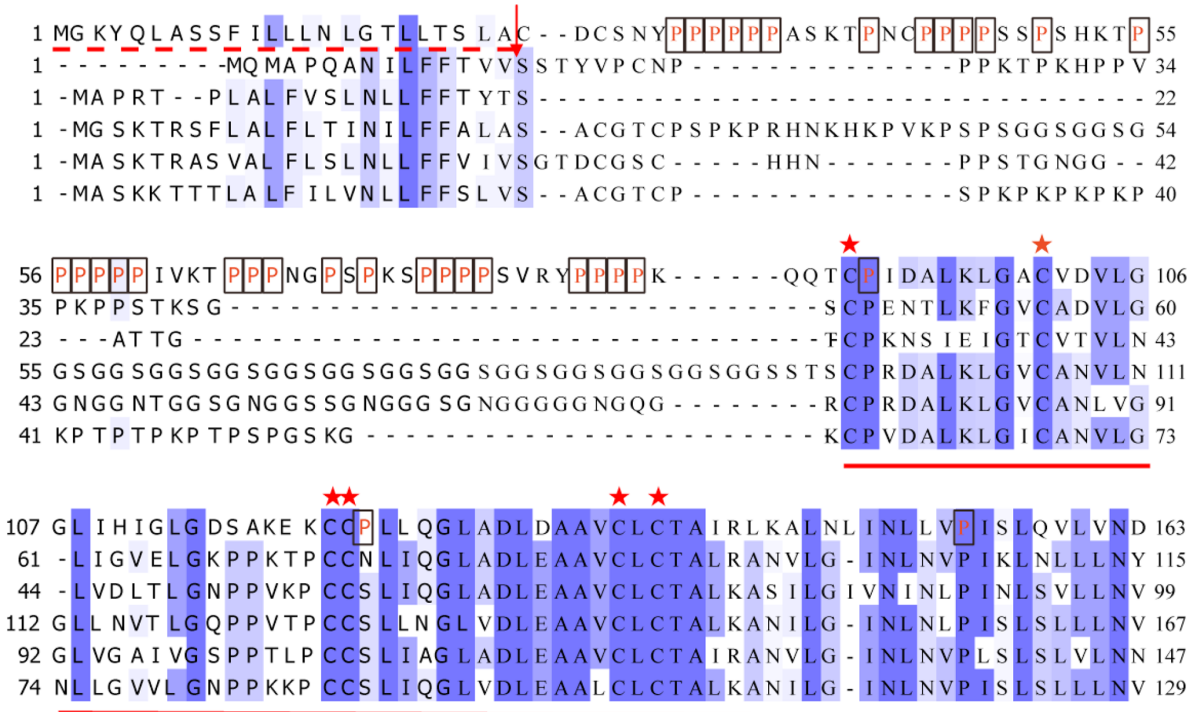

164 CGKY P A G Q Q

116 CG KKT PKDF VCY - 127

100 CSRNAP KS FQCA - 111

168 CSRNAP RDFQCA - 179

148 CGRNPPTGFTC - 158

130 CG KKVP SGFQCPN 142

FIGURE 1 | Sequence alignment between PtrPRP and HyPRPs from other plants. Prolines (P) residues in PtrPRP are marked in red letter and boxed. The signal peptide is indicated with dotted red line at the $\mathrm{N}$-terminal, and the arrow indicates the cleavage site. Identical and highly conserved residues are shaded in dark and light blue, respectively. The conserved $8 \mathrm{CM}$ domain at the C-terminal is underlined, while the cysteine residues are marked by asterisks. GenBank accession numbers for the HyPRPs are AGW00930.1 (PtrPRP, Poncirus trifoliata), AAC49369 (Phaseolous vulgaris), AAM63191.1 (Arabidopsis thaliana), AAM75351.1 (Glycine max), BAA95941 (Nicotiana tabacum), ABQ88334 (C. annuum). 


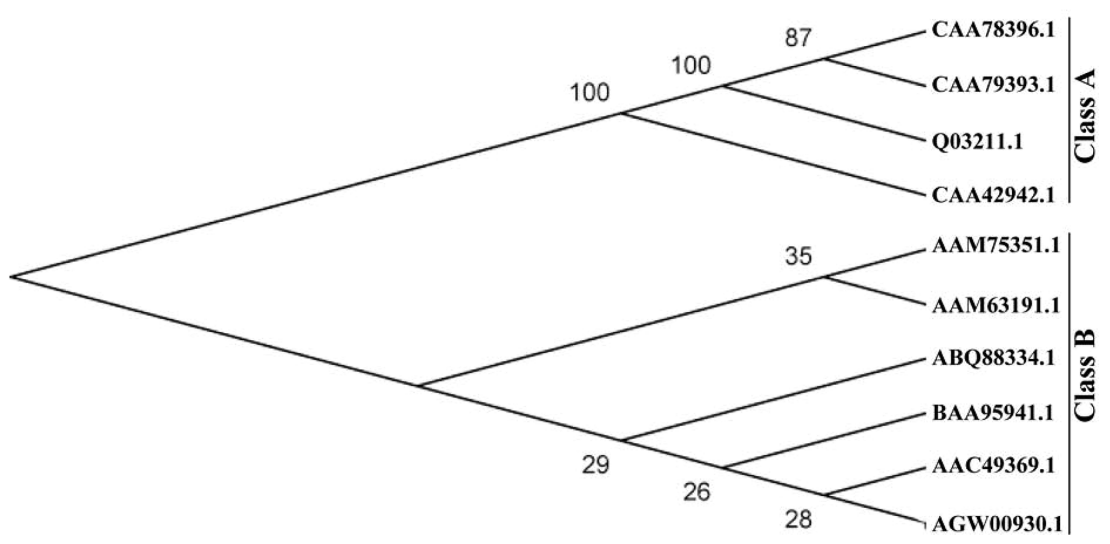

FIGURE 2 | A phylogenetic tree constructed using proline-rich proteins (PRPs) of trifoliate orange and other plants, including CAA78396.1 (N. tabacum), CAA78393.1 (N. tabacum), Q03211.1 (N. tabacum), CAA42942.1 (Phaseolus vulgaris), AGW00930.1 (PtrPRP, Poncirus trifoliata), AAC49369 (Phaseolous vulgaris), AAM63191.1 (A. thaliana), AAM75351.1 (G. max), BAA95941 (N. tabacum), and ABQ88334 (C. annuum). The neighbor-joining tree was generated with MEGA4.0 from 1000 bootstrap replicates.

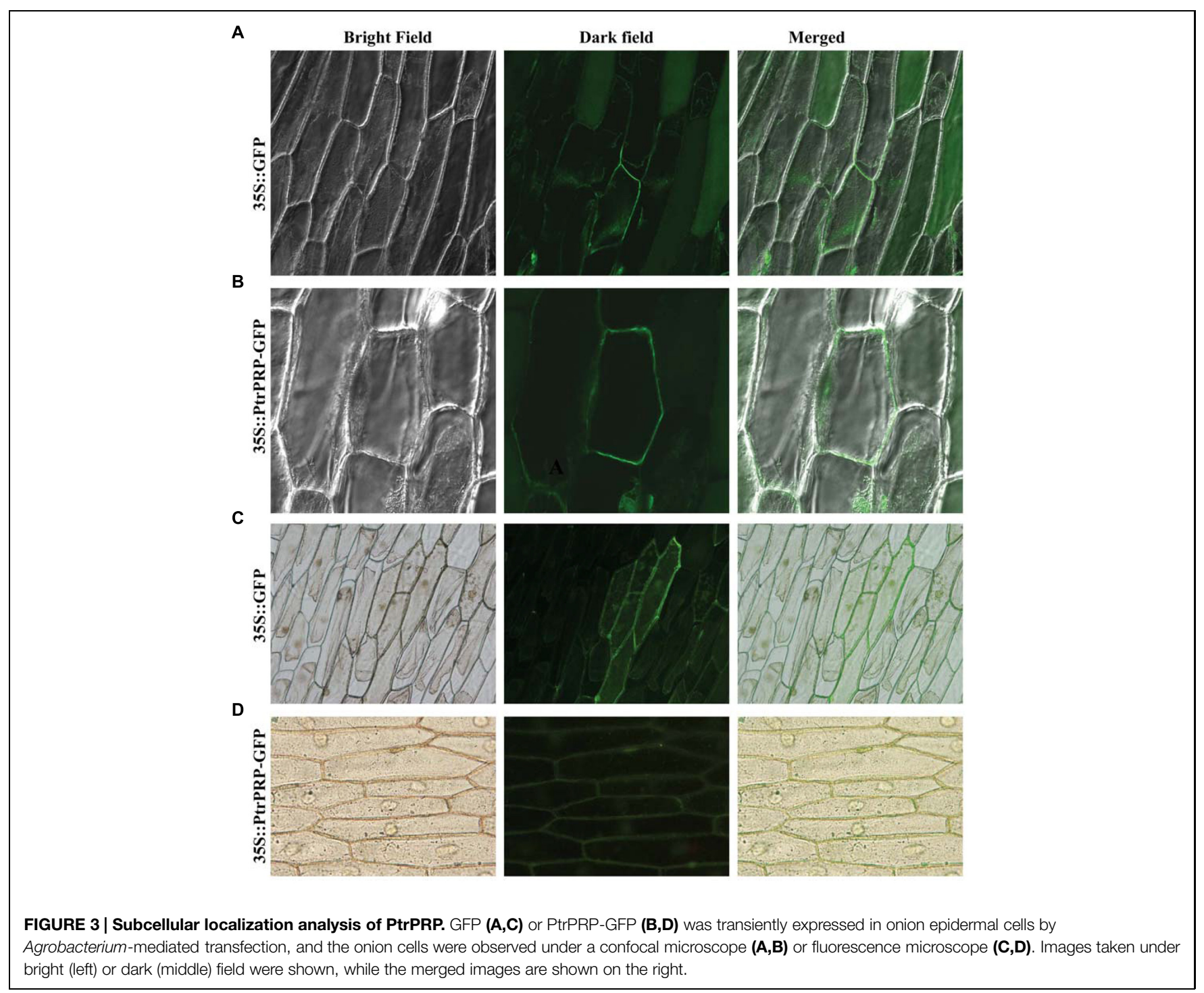


terminal (Figure 1), which is an important signature for a HyPRP, indicating that PtrPRP was actually a typical HyPRP. Besides, the phylogenetic tree indicated that PtrPRP was a Class B HyPRP (Figure 2).

\section{Subcellular Localization of PtrPRP}

Subcellular localization of PtrPRP was investigated by constructing a fusion protein of PtrPRP without the stop codon and GFP, driven by $C a M V 35 S$ promoter, using the GFP vector as a control. Microscopic observation showed that green fluorescence could be observed in the whole cells when the control plasmid was transiently expressed (Figures 3A,C). However, when the fusion protein was expressed in the onion epidermis, green fluorescence was predominantly observed on the outer surface of the cells (Figures 3B,D), which may include the plasma membrane (PM), internal membranes and $\mathrm{PM} /$ cell wall interphase. However, the exact localization to a certain position of PtrPRP remains to be determined.

\section{Expression Profiles of PtrPRP in Different Tissues and Under Various Treatments}

Spatial expression of PtrPRP in three tissues, leaf, root, and stem, was assessed by qPCR. PtrPRP was constitutively expressed in the three tissues, but their transcript levels varied among each other (Figure 4). The highest mRNA abundance was detected in the leaf, whereas root showed the lowest expression level among the three tissues. In addition, expression patterns of PtrPRP in response to cold, dehydration, salt, and $\mathrm{ABA}$ treatments were examined. Under exposure to cold, transcript level of PtrPRP was quickly induced within $6 \mathrm{~h}$, and then progressively accumulated to reach the highest level at $144 \mathrm{~h}$, when the expression level was elevated by more than 30 -folds compared with that at $0 \mathrm{~h}$. However, when the cold treatment was removed, transcript level of $P \operatorname{tr} P P R$ was sharply decreased (Figure 5A). Dehydration treatment led to gradual reduction of PtrPRP mRNA levels up to the lowest value at $3 \mathrm{~h}$, followed by an accretion to the basal level at $6 \mathrm{~h}$. Surprisingly, rehydration for $6 \mathrm{~h}$ resulted in a notable induction of PtrPRP (Figure 5B). Steady-state mRNA level of $\operatorname{Ptr} P R P$ was up-regulated by nearly 14 -folds within $6 \mathrm{~h}$ of salt treatment, and continued to rise until reaching the peak value at $24 \mathrm{~h}$, followed by a sharp decrease to basal level at 72 and $144 \mathrm{~h}$. Relief of the salt stress for $6 \mathrm{~h}$ led to a sevenfolds elevation of the transcript level (Figure 5C). Expression pattern of PtrPRP in response to exogenous $\mathrm{ABA}$ application was similar to that under salt treatment, except the greater induction within the first two time points. Removal of ABA also led to an up-regulation of $P \operatorname{tr} P R P$ transcript (Figure 5D).

\section{Production of Trifoliate Orange RNAi Lines with Knock-down of PtrPRP}

To elucidate the role of PtrPRP in cold tolerance, RNAi strategy was used to suppress PtrPRP in trifoliate orange. For this purpose, a 253-bp cDNA fragment displaying lower degree of sequence conservation among C-terminal ends of the PRPs was used to construct PtrPRP-RNAi vector, which was then transferred into trifoliate orange via Agrobacterium-mediated transformation (Figures 6A-D). Kanamycin-resistant plants were confirmed to be positive using genomic PCR (Figure 6E). Semi-quantitative RT-PCR assay indicated that among the positive lines PtrPRP was successfully down-regulated in three lines, and the greatest suppression was observed in lines $\# 51$ and $\# 52$, which were hereafter designated as RNAi-51 and RNAi-52, respectively (Figure 6F). Expression of PtrPRP in RNAi-51 was also checked using qRT-PCR, and the results confirmed that PtrPRP is trully knocked down, purporting the semi-quantitative RT-PCR data (Figure 6G). The two RNAi lines, RNAi-51 and RNAi-52, showed no difference in plant morphology in comparison with the WT.

\section{Knock-down of PtrPRP Confers Sensitivity to Chilling Stress}

The two RNAi lines and WT were subjected to a chilling temperature at $0^{\circ} \mathrm{C}$ for $48 \mathrm{~h}$ so as to investigate the impact of silencing PtrPRP on cold tolerance. We examined EL and MDA, two critical parameters that have been widely used for evaluating the stress tolerance in earlier studies (Peng et al., 2012, 2014). After the chilling treatment, EL and MDA of both RNAi lines were significantly higher than those of WT, indicating that more severe damages have been imposed on the RNAi lines compared to the WT (Figures 7A,B).

\section{The RNAi Lines Accumulate More ROS but Less Proline}

Electrolyte leakage and MDL are indirect indices for oxidative stress that is primarily caused by excessive accumulation of ROS. The drastic difference in EL and MDA levels between RNAi lines and WT prompted us to check ROS status of the tested lines after cold treatment. We first used histochemical staining with DAB and NBT to reveal in situ accumulation of $\mathrm{H}_{2} \mathrm{O}_{2}$ and $\mathrm{O}_{2}{ }^{-}$, respectively, in the cold treated leaves. This method is valid as the ROS levels can be directly disclosed based on the color of reaction. As shown in Figure 8A, conspicuous difference in the staining patterns was observed between WT and the RNAi lines. The leaves of RNAi lines were stained by both DAB and NBT in deeper manner or the areas of staining were larger, indicating that the RNAi lines produced more ROS after cold treatment compared with the WT. The staining was partly confirmed by quantitative measurement of $\mathrm{H}_{2} \mathrm{O}_{2}$ using a specific kit designed for it (Figure 8B). We also measured proline contents in the RNAi lines and WT after cold treatment, as this compound has been considered as an important metabolite indicating the relevance to stress tolerance. As shown in Figure 9, the WT accumulated more proline in comparison with the two RNAi lines.

\section{Discussion}

Although HyPRPs have been reported in many plants, the precise functions of HyPRPs were still poorly elucidated, especially in woody plants. Here we report molecular cloning and functional characterization of $\operatorname{Ptr} P R P$, a $H y P R P$ gene from Poncirus trifoliata. Since genome of $P$. trifoliata has not been sequenced, it remains to be determined whether $P$. trifoliata has more PRP members at this stage. By search against the whole 


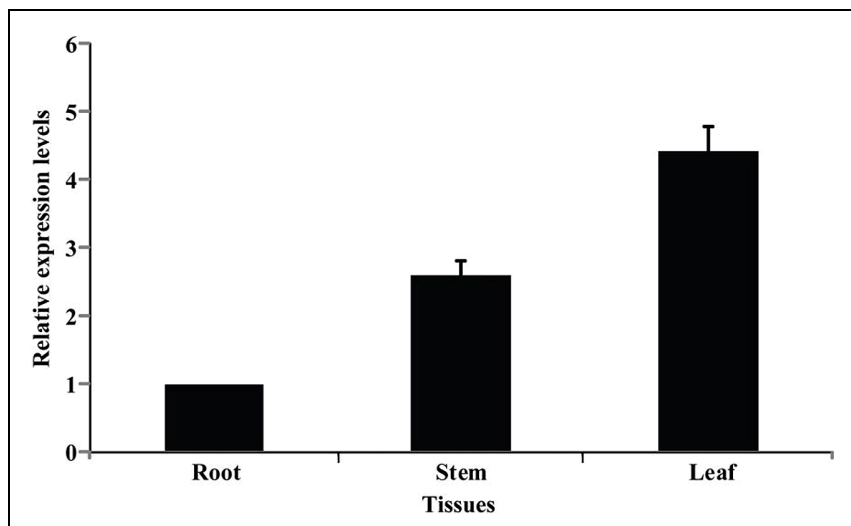

FIGURE 4 | Expression analysis of PtrPRP in three different tissues, including root, stem, and leaf, as revealed by qPCR. Expression level of PtrPRP in the root was set as 1, and those in other tissues were calculated accordingly.

genome sequences of Citrus sinensis, a closely related species of P. trifoliata we found four putative PRPs (CsPRPs), among which CsPRP1 shares the highest similarity with PtrPRP. It suggests that P. trifoliata may possibly contain other PRPs, but this assumption needs to be verified in the future. In PtPRP, proline residues account for $21 \%$ of the total amino acids of PtrPRP, allowing us to believe that PtrPRP is a PRP. Of note, the prominent abundance of proline residues at the $\mathrm{N}$ terminus may be possibly associated with the targeting of this protein, as the presence of hydrophobic proline-rich (HPR) motif has been suggested to be necessary and sufficient for the intracellular targeting of a temperature-induced lipocalin in Arabidopsis (AtTIL; Hernaindez-Gras and Boronat, 2015). So far, PRPs have been previously categorized into five groups based on signature motifs (Gothandam et al., 2010), but PtrPRP belongs to none of them. However, sequence alignments between PtrPRP and HyPRPs from several plants revealed that PtrPRP has the conserved $8 \mathrm{CM}$ domain, which is a typical signature of HyPRPs, but not present in PRPs (Josè-Estanyol et al., 2004), which indicates that PtrPRP is possibly considered as a HyPRP. In addition, since PtrPRP contains the $8 \mathrm{CM}$ domain it should be categorized into the Class B HyPRPs.

Expression of PRPs was shown to be associated with development of various tissues and exhibited temporal and spatial expression patterns (Vignols et al., 1999; Gothandam et al., 2010; Neto et al., 2013). Transcripts of $Z m H y P R P$ from maize were specifically observed in the immature embryos (JosèEstanyol et al., 1992), while a $H y P R P$ gene of strawberry was exclusively found in mature fruits (Blanco-Portales et al., 2004). Hong et al. (1989) reported that soybean contained three PRPs, SbPRP1, SbPRP2, and SbPRP3. SbPRP1 was highly expressed in mature hypocotyl, root, and immature seed coat. $S b P R P 2$ was the predominant form of transcripts in the apical hypocotyl and young suspension culture cells, while the transcripts of SbPRP3 accumulated mainly in aerial parts, especially in leaves. In a recent study, Neto et al. (2013) expanded the soybean HyPRP proteins to 35 members, which exhibited variable expression patterns in six vegetative organs, root, and root tip, nodule, leaves, green pods, flower, and apical meristem. Some of them
A

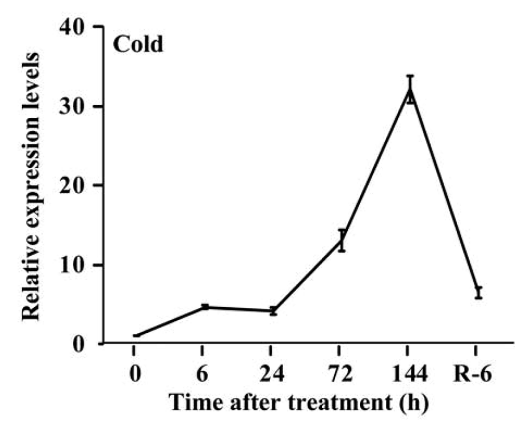

C

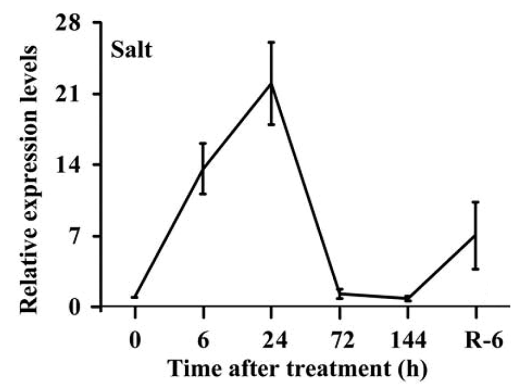

B
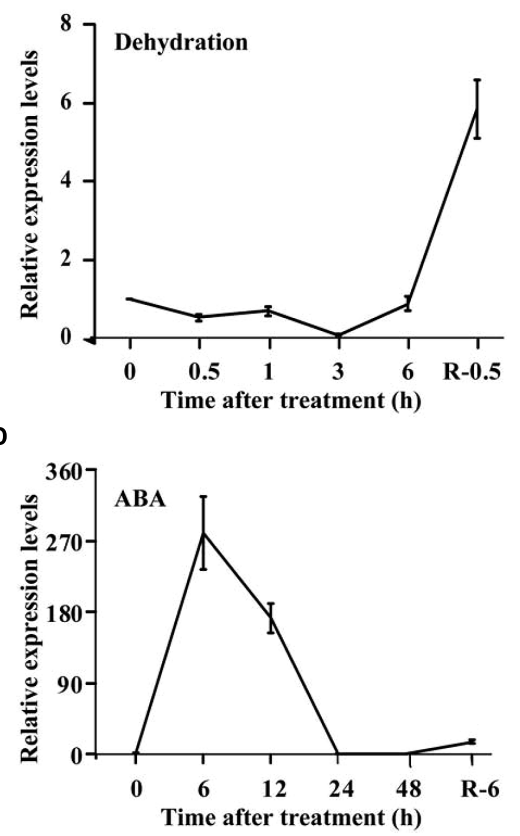

FIGURE 5 | Expression analysis of PtrPRP in response to various treatment, including cold (A), dehydration (B), salt (C), and ABA (D). Expression levels of PtrPRP at the onset of treatment $(0 \mathrm{~h})$ were set as 1 , and those at other time points were calculated accordingly. R indicates recovery after the corresponding treatments. 
A

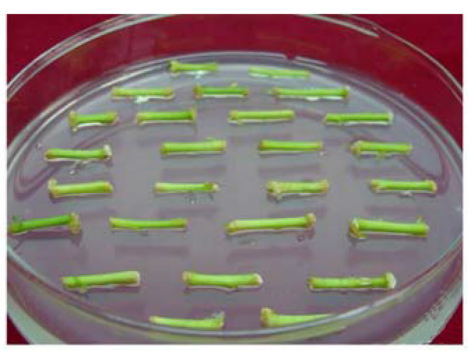

C

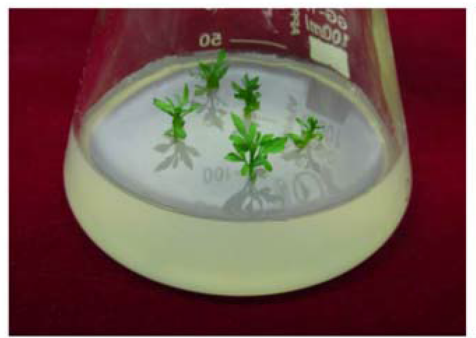

E M P - 3839404142434445464748495051525354
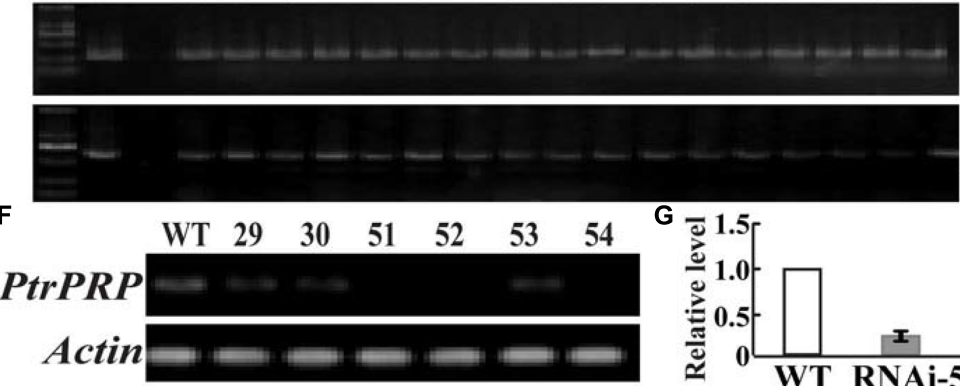
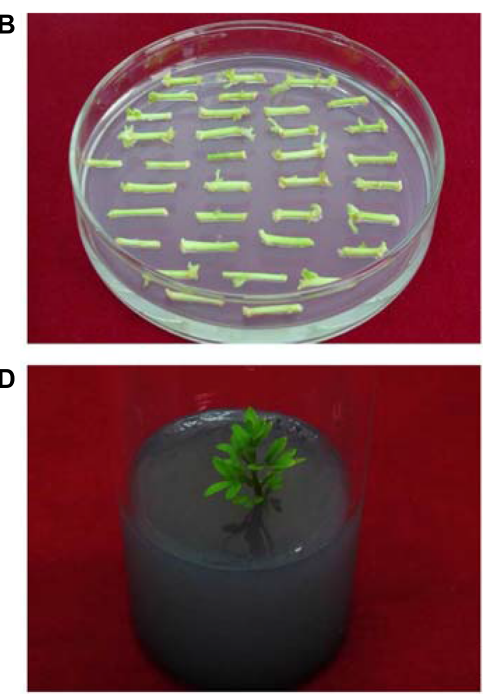

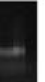
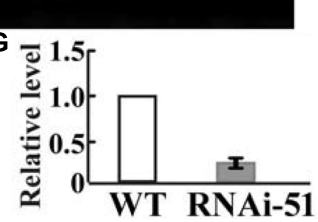

FIGURE 6 | Transformation, regeneration, and characterization of trifoliate orange transgenic plants. (A,B) Culture of the stem segments on selection medium for $30 \mathrm{~d}(\mathbf{A})$ and $60 \mathrm{~d}(\mathbf{B})$, respectively. (C) Regeneration of kanamycin -resistant shoots on the selection medium. (D) A rooting plant on the root-inducing medium. (E) Genomic PCR of the kanamycin-resistant plants using designed primers specific to PtrPRP (upper) and NPTII (bottom), respectively. (F) Expression analysis of PtrPRP in six positive transgenic plants, as revealed by RT-PCR. Actin gene was used as an internal control. (G) Analysis of PtrPRP expression level in RNAi-51 using qRT-PCR.

were not detected in any tissue, while others were expressed in specific organs. Interestingly, four soybean $H y P R P s$ were almost exclusively highly expressed in leaves, consistent with our finding on PtrPRP, which displayed the highest expression level in leaf. These findings suggest that plant PRPs may exhibit spatial expression patterns, which implies that the PRPs may function in different ways among various tissues.

In this study, we also noticed that PtrPRP was remarkably induced by cold and salt, but underwent minor change under dehydration. Of note, when the abiotic stresses were removed, PtrPRP showed opposite expression patterns in comparison with those under the corresponding stresses. These results indicate that PtrPRP was sensitively responsive to the stresses, but the response is different among various environmental stimuli. This phenomenon has been also observed in other PRP gene. For example, OsPRP1 exhibited different or even reverse expression patterns under various stress factors (Wang et al., 2006). In another work, CcHyPRP transcripts of pigeonpea (Cajanus cajan L.) were shown to be enhanced in response to treatments with PEG, $\mathrm{NaCl}$, heat $\left(42^{\circ} \mathrm{C}\right)$, and cold (Priyanka et al., 2010). These findings suggest that plant PRP genes are differentially regulated under abiotic stresses and that different member of the PRP family may possibly play specific roles in mediating abiotic stress tolerance. In addition, PtrPRP was quickly and sharply induced by ABA, implying that PtrPRP is an ABA-responsive gene. However, expression of PtrPRP under ABA treatment was consistent with RePRP of rice and $C c H y P R P$ of pigeonpea, but it was contradictory to OsPRP, which was repressed by ABA (Akiyama and Pillai, 2003; Priyanka et al., 2010; Tseng et al., 2013). However, whether PtrPRP functions in an ABA-dependent manner needs to be elucidated.

Down-regulation of gene expression via RNAi at posttranscriptional level has been widely used as an alternative approach for functionally characterizing genes involved in biotic and abiotic stress tolerance, because RNAi plants may clearly display altered phenotype or metabolic disorders (Mao et al., 2007; Tardieu and Tuberosa, 2010; Duan et al., 2012; Huang et al., 2013; Saurabh et al., 2014). In keeping with this, herein we employed RNAi approach to knock down PtrPRP in trifoliate orange so as to elucidate the function of this gene in cold 


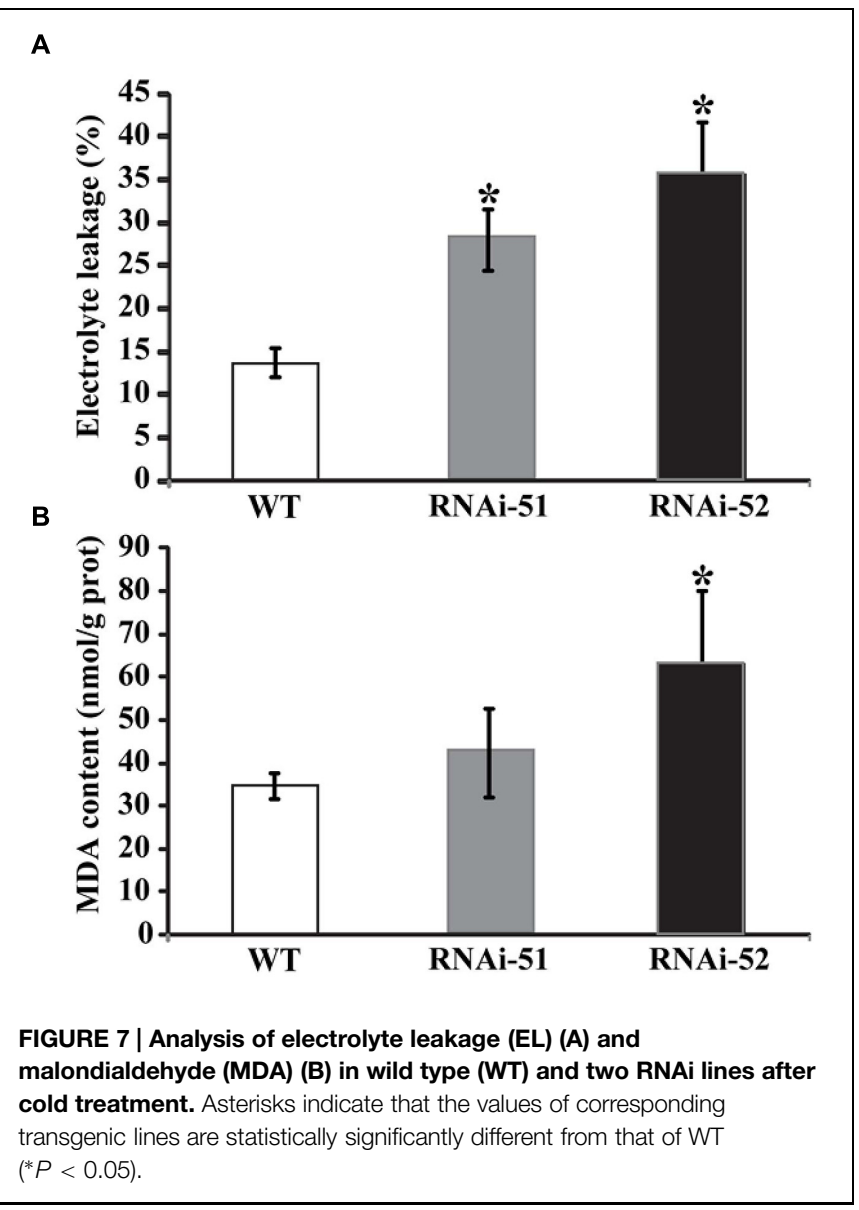

stress tolerance. After $48 \mathrm{~h}$ of cold treatment, EL and MDA content, two parameters for membrane integrity, in the RNAi lines were higher than in the control, suggesting that knock-down of PtrPRP led to severer membrane damage. It was reasonable because PtrPRP was localized in the membrane. In higher plant, cell membrane encloses cytoplasm and various organelles; thus maintenance of cell membrane integrity is critical for plant to overcome the physiological and biochemical changes induced by cold stress. The well-documented injuries caused by cold are largely due to osmotic and oxidative stresses that pose serious threat to membrane integrity (Welti et al., 2002; Peng et al., 2014). Therefore, when challenged by low temperature stress, biosynthesis and degradation of some related proteins might be expedited to stabilize the integrity of cellular membranes against cold injury (Achard et al., 2008). PRPs are proposed to play an integral role in consolidation of extracellular matrix structure of plant cells, which is an important approach for elevating mechanical strength to the cell wall. In this case, the PRPs may confer the integrity of plant membranes and promote the structure maintenance of organs (Gothandam et al., 2010). Therefore, it is reasonable to assume that when this protein accumulation was suppressed by RNAi, the protective roles of PtrPRP in membrane integrity might be impaired, which in turn leads to greater membrane damage, as revealed by higher EL and MDA levels.

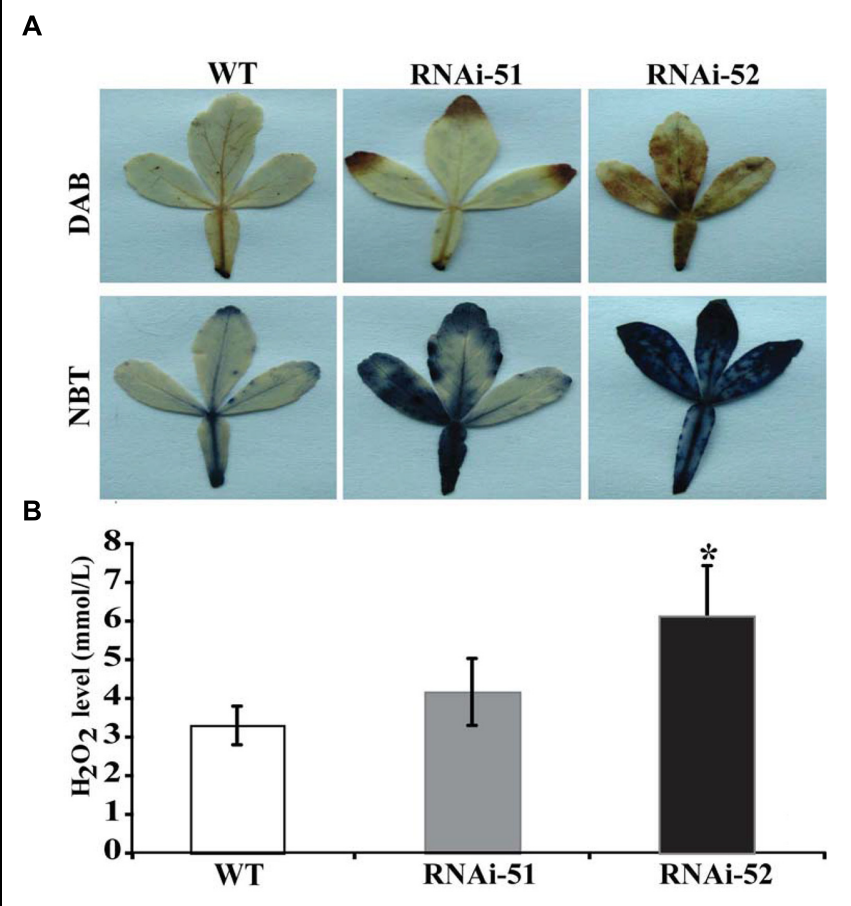

FIGURE 8 | Histochemical staining and quantitative measurement of ROS. (A) Histochemical staining with DAB (upper) and NBT (lower) in WT and two RNAi lines after cold treatment. (B) Quantitative measurement of $\mathrm{H}_{2} \mathrm{O}_{2}$ in WT and the RNAi lines. Asterisks indicate that the values of corresponding transgenic lines are statistically significantly different from that of WT $\left({ }^{*} P<0.05\right)$.

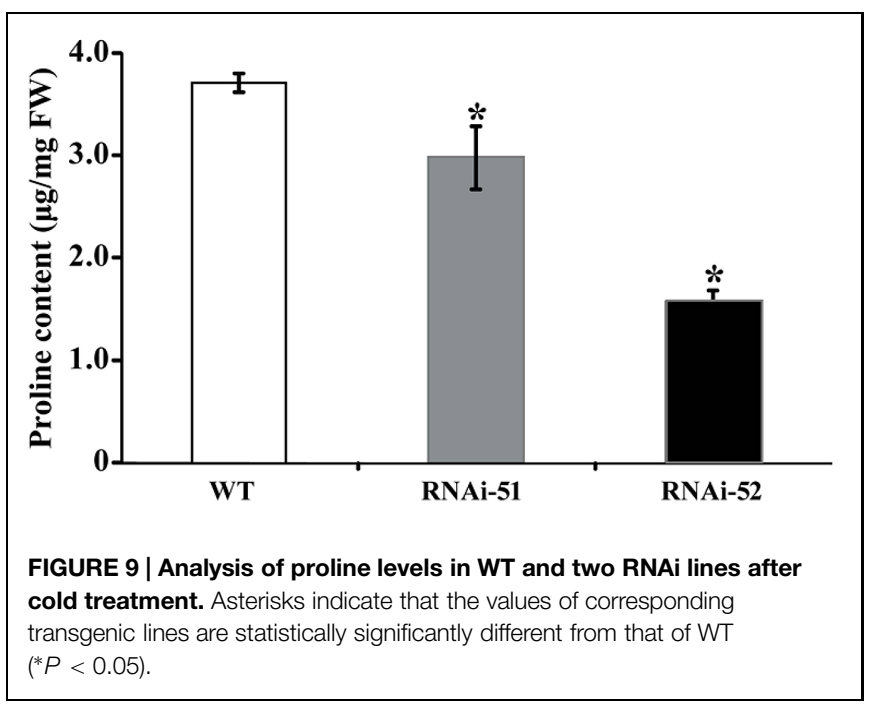

Proline has been documented to act as an important compounds involved in stress tolerance as they can serve as an osmolyte to optimize the physical structure of cell membrane for proper cellular function (Duncan and Widholm, 1987; Akiyama and Pillai, 2003; Rodriguez and Redman, 2005; Battaglia et al., 2007). The proline level has been shown to be associated with the magnitude of a plant to alleviate membrane damages and enhance 
cell viability via mitigation of osmotic stress and reduction of MDA production (Ozden et al., 2009), which agrees with our data that MDA content in the two RNAi lines was higher than in the WT. These illustrations suggest that accumulation of less proline may lead to disordered osmotic adjustment, which constitutes a mechanism underlying the cold sensitivity of the RNAi lines. Except acting as an osmolyte, PtrPRP may also contribute to scavenging of ROS, which are by-products of various metabolic processes (Mittler, 2002; Finkel, 2011). Excessive accumulation of ROS is known to cause oxidative damages to cells and thus impairs normal physiological or biological processes. Plant has developed either enzymatic or non-enzymatic antioxidant defense systems to maintain ROS homeostasis and to alleviate the burst of ROS. The non-enzymatic system is composed of a number of compounds that are generally known as low MW antioxidants, such as betaine and proline (Rodriguez and Redman, 2005; Fu et al., 2011; Hayat et al., 2012; Keunen et al., 2013; Peng et al., 2014). In earlier studies, exogenous application of proline has been shown to mitigate ROS production and confer enhanced stress tolerance, implying that proline plays a significant role in ROS scavenging (Hoque et al., 2007; Kaul et al., 2008). Hong et al. (2000) suggested that the role of proline as a ROS scavenger is more important than its role as an osmolyte under stress conditions, further supporting the crucial value of proline in detoxifying ROS. In this work, we found that suppression of PtrPRP in the RNAi lines was accompanied by a noticeable reduction of proline, concurrent with the prominent

\section{References}

Achard, P., Gong, F., Cheminant, S., Alioua, M., Hedden, P., and Genschik, P. (2008). The cold-inducible CBF1 factor-dependent signaling pathway modulates the accumulation of the growth-repressing DELLA proteins via its effect on gibberellin metabolism. Plant Cell 20, 2117-2129. doi: 10.1105/tpc.108.058941

Akiyama, T., and Pillai, M. A. (2003). Isolation and characterization of a gene for a repetitive proline rich protein (OsPRP) down regulated during submergence in rice (Oryza sativa). Physiol. Plant. 118, 507-513. doi: 10.1034/j.13993054.2003.00104.X

Bannai, H., Tamada, Y., Maruyama, O., Nakai, K., and Miyano, S. (2002). Extensive feature detection of N-terminal protein sorting signals. Bioinformatics 18, 298-305. doi: 10.1093/bioinformatics/18.2.298

Battaglia, M., Soloìrzano, R. M., Hernaìndez, M., Cueillar-Ortiz, S., GarciìaGoìmez, B., Maìrquez, J., et al. (2007). Proline-rich cell wall proteins accumulate in growing regions and phloem tissue in response to water deficit in common bean seedlings. Planta 225, 1121-1133. doi: 10.1007/s00425-006-0423-9

Blanco-Portales, R., Lopez-Raez, J. A., Bellido, M. L., Moyano, E., Dorado, G., Gonzalez-Reyez, J. A., et al. (2004). A strawberry fruit-specific and ripeningrelated gene codes for a HyPRP protein involved in polyphenol anchoring. Plant Mol. Biol. 55, 763-780. doi: 10.1007/s11103-005-1966-Z

Bradford, M. M. (1976). A rapid and sensitive method for the quantitation of microgram quantities of protein utilizing the principle of protein-dye binding. Anal. Biochem. 72, 248-254. doi: 10.1016/0003-2697(76)90527-3

Bouton, S., Viau, L., Lelièvre, E., and Limami, A. M. (2005). A gene encoding a protein with a proline-rich domain (MtPPRD1), revealed by suppressive subtractive hybridization (SSH), is specifically expressed in the Medicago truncatula embryo axis during germination. J. Exp. Bot. 56, 825-832. doi: 10.1093/jxb/eri077

Dereeper, A., Guignon, V., Blanc, G., Audic, S., Buffet, S., Chevenet, F., et al. (2008). Phylogeny.fr: robust phylogenetic analysis for the non-specialist. Nucleic Acids Res. 36, W465-W469. doi: 10.1093/nar/gkn180 elevation of ROS levels. These findings indicate that PtrPRP may function in cold tolerance by influencing ROS homeostasis due to, at least in part, the role of proline as an efficient antioxidant for ROS detoxification. Nevertheless, why and how knock-down of PtrPRP caused a decrease of proline in the RNAi lines remained elusive and needs to be investigated in the future.

\section{Conclusion}

Our data demonstrated that PtrPRP of $P$. trifoliata is a hybridPRP. PtrPRP expresses in various tissues and responds to abiotic stimuli, such as cold, salt, and ABA. RNAi-based knock-down of PtrPRP conferred enhanced cold sensitivity of the RNAi lines at chilling temperature, as manifested by severer membrane damage, accumulation of more ROS and less production of proline under cold stress. Therefore, PtrPRP may contribute to cold tolerance via modulation of proline, an important osmolyte and ROS scavenger.

\section{Acknowledgments}

This work was financially supported by the National Science Foundation of China, the Ministry of Agriculture, and the National High Technology Research and Development Program of China (2011AA100205).

Deutch, C. E., and Winicov, I. (1995). Post-transcriptional regulation of a salt-inducible alfalfa gene encoding a putative chimeric proline-rich cell wall protein. Plant Mol. Biol. 27, 411-418. doi: 10.1007/BF000 20194

Duan, C. G., Wang, C. H., and Guo, H. S. (2012). Application of RNA silencing to plant disease resistance. Science 3:5. doi: 10.1186/1758-907X-3-5

Duncan, D. R., and Widholm, J. M. (1987). Proline accumulation and its implication in cold tolerance of regenerable maize callus. Plant Physiol. 83, 703-708. doi: 10.1104/pp.83.3.703

Dvořáková, L., Cvrčková, F., and Fischer, L. (2007). Analysis of the hybrid prolinerich protein families from seven plant species suggests rapid diversification of their sequences and expression patterns. BMC Genomics 8:412. doi: 10.1186/1471-2164-8-412

Dvořáková, L., Srba, M., Opatrny, Z., and Fischer, L. (2012). Hybrid proline-rich proteins: novel players in plant cell elongation? Ann. Bot. 109, 453-462. doi: $10.1093 / \mathrm{aob} / \mathrm{mcr} 278$

Einset, J., Winge, P., and Bones, A. (2007). ROS signaling pathways in chilling stress. Plant Signal. Behav. 2, 365-367. doi: 10.4161/psb.2. 5.4461

Finkel, T. (2011). Signal transduction by reactive oxygen species. J. Cell Biol. 194, 7-15. doi: 10.1083/jcb.201102095

Fowler, T. J., Bernhardt, C. B., and Tierney, M. L. (1999). Characterization and expression of four proline-rich cell wall proteins in Arabidopsis encoding two distinct subsets of multiple domain proteins. Plant Physiol. 121, 1081-1091. doi: 10.1104/pp.121.4.1081

Fu, X. Z., Khan, E. U., Hu, S. S., Fan, Q. J., and Liu, J. H. (2011). Overexpression of the betaine aldehyde dehydrogenase gene from Atriplex hortensis enhances salt tolerance in the transgenic trifoliate orange (Poncirus trifoliata (L.) Raf.). Environ. Exp. Bot. 74, 106-113. doi: 10.1016/j.envexpbot.2011.05.006

Gothandam, K. M., Nalini, E., Karthikeyan, S., and Shin, J. S. (2010). OsPRP3, a flower specific proline-rich protein of rice, determines extracellular matrix structure of floral organs and its overexpression confers cold-tolerance. Plant Mol. Biol. 72, 125-135. doi: 10.1007/s11103-009-9557-z 
Hayat, S., Hayat, Q., Alyemeni, M. N., Wani, A. S., Pichtel, J., and Ahmad, A. (2012). Role of proline under changing environments. Plant Signal. Behav. 7, 1456-1466. doi: 10.4161/psb.21949

He, C. Y., Zhang, J. S., and Chen, S. Y. (2002). A soybean gene encoding a proline-rich protein is regulated by salicylic acid, an endogenous circadian rhythm and by various stresses. Theor. Appl. Genet. 104, 1125-1131. doi: 10.1007/s00122-001-0853-5

Hernaindez-Gras, F., and Boronat, A. (2015). A hydrophobic proline-rich motif is involved in the intracellular targeting of temperature-induced lipocalin. Plant Mol. Biol. 88, 301-311. doi: 10.1007/s11103-015-0326-x

Holk, A., Klumpp, L., and Scherer, G. F. E. (2002). A cell wall protein downregulated by auxin suppressed cell expansion in Daucus carota (L.). Plant Mol. Biol. 50, 295-305. doi: 10.1023/A:1016052613196

Hong, J. C., Nagao, R. T., and Key, J. L. (1989). Developmentally regulated expression of soybean proline-rich cell wall protein genes. Plant Cell 1, 937-943. doi: $10.1105 /$ tpc.1.9.937

Hong, Z., Lakkineni, K., Zhang, Z., and Verma, D. P. (2000). Removal of feedback inhibition of delta(1)-pyrroline-5-carboxylate synthetase results in increased proline accumulation and protection of plants from osmotic stress. Plant Physiol. 122, 1129-1136. doi: 10.1104/pp.122.4.1129

Hoque, M. A., Banu, M. N., Okuma, E., Amako, K., Nakamura, Y., Shimoishi, Y., et al. (2007). Exogenous proline and glycinebetaine increase $\mathrm{NaCl}$-induced ascorbate-glutathione cycle enzyme activities, and proline improves salt tolerance more than glycinebetaine in tobacco Bright Yellow-2 suspensioncultured cells. J. Plant Physiol. 164, 1457-1468. doi: 10.1016/j.jplph.2006. 10.004

Huang, X. S., Luo, T., Fu, X. Z., Fan, Q. J., and Liu, J. H. (2011). Cloning and molecular characterization of a mitogen-activated protein kinase gene from Poncirus trifoliata whose ectopic expression confers dehydration/drought tolerance in transgenic tobacco. J. Exp. Bot. 62, 51915206. doi: 10.1093/jxb/err229

Huang, X. S., Wang, W., Zhang, Q., and Liu, J. H. (2013). A basic Helix-Loop-Helix transcription factor, PtrbHLH, of Poncirus trifoliata confers cold tolerance and modulates peroxidase-mediated scavenging of hydrogen peroxide. Plant Physiol. 162, 1178-1194. doi: 10.1104/pp.112.210740

Josè-Estanyol, M., Gomis-Rüth, F. X., and Puigdomènech, P. (2004). The eightcysteine motif, a versatile structure in plant proteins. Plant Physiol. Biochem. 42, 355-365. doi: 10.1016/j.plaphy.2004.03.009

Josè-Estanyol, M., and Puigdomènech, P. (2000). Plant cell wall glycoproteins and their genes. Plant Physiol. Biochem. 38, 97-108. doi: 10.1016/S09819428(00)00165-0

Josè-Estanyol, M., Ruiz-Avila, L., and Puigdomènech, P. (1992). A maize embryospecific genes encode a proline-rich and hydrophobic protein. Plant Cell 4, 413-423. doi: 10.2307/3869443

Kaul, S., Sharma, S. S., and Mehta, I. K. (2008). Free radical scavenging potential of L-proline: evidence from in vitro assays. Amino Acids 34, 315-320. doi: 10.1007/s00726-006-0407-x

Keunen, E., Peshev, D., Vangronsveld, J., Van Den Ende, W., and Cuypers, A. (2013). Plant sugars are crucial players in the oxidative challenge during abiotic stress: extending the traditional concept. Plant Cell Environ. 36, 1242-1255. doi: $10.1111 /$ pce. 12061

Knight, M. R., Trewavas, A. J., and Knight, M. R. (1996). Cold calcium signaling in Arabidopsis involves two cellular pools and a change in calcium signature after acclimation. Plant Cell 8, 489-503. doi: 10.1105/tpc.8.3.489

Mao, Y. B., Cai, W. J., Wang, J. W., Hong, G. J., Tao, X. Y., Wang, L. J., et al. (2007). Silencing a cotton bollworm P450 monooxygenase gene by plant mediated RNAi impairs larval tolerance of gossypol. Nat. Biotechnol. 25, 1307-1313. doi: $10.1038 /$ nbt 1352

Mittler, R. (2002). Oxidative stress, antioxidants and stress tolerance. Trends Plant Sci. 7, 405-410. doi: 10.1016/S1360-1385(02)02312-9

Nakashima, K., and Yamaguchi-Shinozaki, K. (2006). Regulons involved in osmotic stress-responsive and cold stress-responsive gene expression in plants. Physiol. Plant. 126, 62-71. doi: 10.1111/j.1399-3054.2005. 00592.x

Neto, L. B., de Oliveira, R. R., Wiebke-Strohm, B., Bencke, M., Weber, R. L., Cabreira, C., et al. (2013). Identification of the soybean HyPRP family and specific gene response to Asian soybean rust disease. Genet. Mol. Biol. 36, 214-224. doi: 10.1590/S1415-47572013005000017
Ozden, M., Demirelb, U., and Kahramanb, A. (2009). Effects of proline on antioxidant system in leaves of grapevine (Vitis vinifera L.) exposed to oxidative stress by $\mathrm{H}_{2} \mathrm{O}_{2}$. Sci. Hortic. 119, 163-168. doi: 10.1016/j.scienta.2008.07.031

Peng, T., Zhu, X. F., Duan, N., and Liu, J. H. (2014). PtrBAM1, a $\beta$-amylase-coding gene of Poncirus trifoliata, is a CBF regulon member with function in cold tolerance by modulating soluble sugar levels. Plant Cell Environ. 37, 2754-2767. doi: $10.1111 /$ pce. 12384

Peng, T., Zhu, X. F., Fan, Q. J., Sun, P. P., and Liu, J. H. (2012). Identification and characterization of low temperature stress responsive genes in Poncirus trifoliata by suppression subtractive hybridization. Gene 492, 220-228. doi: 10.1016/j.gene.2011.10.025

Priyanka, B., Sekhar, K., Reddy, V. D., and Rao, K. V. (2010). Expression of pigeonpea hybrid-proline-rich protein encoding gene ( $\mathrm{CcHyPRP})$ in yeast and Arabidopsis affords multiple abiotic stress tolerance. Plant Biotechnol. J. 8, 76-87. doi: 10.1111/j.1467-7652.2009.00467.x

Rodriguez, R., and Redman, R. (2005). Balancing the generation and elimination of reactive oxygen species. Proc. Natl. Acad. Sci. U.S.A. 102, 3175-3176. doi: 10.1073/pnas.0500367102

Saurabh, S., Vidyarthi, A. S., and Prasad, D. (2014). RNA interference: concept to reality in crop improvement. Planta 239, 543-564. doi: 10.1007/s00425-0132019-5

Shi, Y., Ding, Y., and Yang, S. (2015). Cold signal transduction and its interplay with phytohormones during cold acclimation. Plant Cell Physiol. 56, 7-15. doi: $10.1093 / \mathrm{pcp} / \mathrm{pcu} 115$

Shi, J., Fu, X. Z., Peng, T., Huang, X. S., Fan, Q. J., and Liu, J. H. (2010). Spermine pretreatment confers dehydration tolerance of citrus in vitro plants via modulation of antioxidative capacity and stomatal response. Tree Physiol. 30, 914-922. doi: 10.1093/treephys/tpq030

Stockinger, E. J., Gilmour, S. J., and Thomashow, F. (1997). Arabidopsis thaliana CBF1 encodes an AP2 domain-containing transcriptional activator that binds to the C-repeat/DRE, a cis-acting DNA regulatory element that stimulates transcription in response to low temperature and waster deficit. Proc. Natl. Acad. Sci. U.S.A. 94, 1035-1040. doi: 10.1073/pnas.94. 3.1035

Tardieu, F., and Tuberosa, R. (2010). Dissection and modelling of abiotic stress tolerance in plants. Curr. Opin. Plant Biol. 13, 206-212. doi: 10.1016/j.pbi.2009.12.012

Tseng, I. C., Hong, C. Y., Yu, S. M., and Ho, T. H. (2013). Abscisic acid- and stressinduced highly proline-rich glycoproteins regulate root growth in rice. Plant Physiol. 163, 118-134. doi: 10.1104/pp.113.217547

Vignols, F., Jośe-Estanyol, M., Caparrós-Ruiz, D., Rigau, J., and Puigdomènech, P. (1999). Involvement of a maize proline-rich protein in secondary cell wall formation as deduced from its specific mRNA localization. Plant Mol. Biol. 39, 1999. doi: 10.1023/A:1006129703262

Walker, D. J., Romero, P., and Correal, E. (2010). Cold tolerance, water relations and accumulation of osmolytes in Bituminaria bituminosa. Biol. Plantarum 54, 293-298. doi: 10.1007/s10535-010-0051-x

Wang, R., Chong, K., and Wang, T. (2006). Divergence in spatial expression patterns and in response to stimuli of tandem repeat paralogues encoding a novel class of proline rich proteins in Oryza sativa. J. Exp. Bot. 57, 2887-2897. doi: 10.1093/jxb/erl057

Welling, A., and Palva, E. T. (2006). Molecular control of cold acclimation in trees. Physiol. Plant. 127, 167-181. doi: 10.1111/j.1399-3054.2006.00672.x

Welti, R., Li, W., Li, M., Sang, Y., Biesiada, H., Zhou, H. E., et al. (2002). Profiling membrane lipids in plant stress responses. Role of phospholipase D alpha in freezing-induced lipid changes in Arabidopsis. J. Biol. Chem. 277, 31994-32002. doi: 10.1074/jbc.M205375200

Wilson, R. C., and Cooper, J. B. (1994). Characterization of PRP1 and PRP2 from Medicago truncatula. Plant Physiol. 105, 445-446. doi: 10.1104/pp.105.1.445

Xiong, L., and Zhu, J. K. (2002). Molecular and genetic aspects of plant responses to osmotic stress. Plant Cell Environ. 25, 131-139. doi: 10.1046/j.13653040.2002.00782.x

Xu, D., Huang, X., Xu, Z. Q., and Schläppi, M. (2011). The HyPRP gene EARLI1 has an auxiliary role for germinability and early seedling development under low temperature and salt stress conditions in Arabidopsis thaliana. Planta 234, 565-577. doi: 10.1007/s00425-011-1425-9

Yamaguchi-Shinozaki, K., and Shinozaki, K. (2005). Organization of cisacting regulatory elements in osmotic- and cold-stress-responsive 
promoters. Trends Plant Sci. 10, 88-94. doi: 10.1016/j.tplants.2004. 12.012

Zarka, D. G., Vogel, J. T., Cook, D., and Thomashow, M. F. (2003). Cold induction of Arabidopsis CBF genes involves multiple ICE (Inducer of CBF Expression) promoter elements and a cold-regulatory circuit that is desensitized by low temperature. Plant Physiol. 133, 910-918. doi: 10.1104/pp.103. 027169

Zhang, I., and Schläppi, M. (2007). Cold responsive EARLI1 type HyPRPs improve freezing survival of yeast cells and form higher order complexes in plants. Planta 227, 233-243. doi: 10.1007/s00425-0070611-2

Zhao, L. N., Liu, F. X., Xu, W. Y., Di, C., Zhou, S. X., Xue, Y. B., et al. (2009). Increased expression of OsSPX1 enhances cold/subfreezing tolerance in tobacco and Arabidopsis thaliana.Plant Biotechnol. J. 7, 550-561. doi: 10.1111/j.1467-7652.2009.00423.x

Conflict of Interest Statement: The authors declare that the research was conducted in the absence of any commercial or financial relationships that could be construed as a potential conflict of interest.

Copyright (c) 2015 Peng, Jia and Liu. This is an open-access article distributed under the terms of the Creative Commons Attribution License (CC BY). The use, distribution or reproduction in other forums is permitted, provided the original author(s) or licensor are credited and that the original publication in this journal is cited, in accordance with accepted academic practice. No use, distribution or reproduction is permitted which does not comply with these terms. 